\title{
Al-powered cloud for COVID-19 and other infectious disease diagnosis
}

\author{
Fadi Al-Turjman ${ }^{1}$
}

(C) The Author(s), under exclusive licence to Springer-Verlag London Ltd., part of Springer Nature 2021

The COVID-19 pandemic has revealed many information gaps in our understanding of the spread of contagious diseases, preventative measures, and the development and deployment of vaccines. The combination of Artificial Intelligence, machine learning, big data, cloud computing, next generation networks, and mobile devices may play a role filling some of these gaps. This special issue features 16 selected papers with high quality. The first article, "A systematic approach for COVID-19 predictions and parameter estimation," attempted to predict and analyze the disease along with its related issues to determine the maximum number of infected people, the speed of spread, and most importantly, its evaluation using a model-based parameter estimation method. In this research, the Susceptible-InfectiousRecovered model with different conditions has been used for the analysis of COVID-19. The effects of lockdown, the light switch method, and parameter variations like contact ratio and reproduction number are also analyzed. The authors have attempted to study and predict the lockdown effect, particularly in India in terms of infected and recovered numbers, which show substantial improvement. A disease-free endemic stability analysis using Lyapunov and LaSalle's method is presented, and novel methods such as the convalescent plasma method and the Who Acquires Infection From Whom method are also discussed, as they are considered to be useful in flattening the curve of COVID-19.

The second article titled "Time series forecasting of COVID-19 transmission in Asia Pacific countries using deep neural networks," utilized the latest deep learning techniques such as Long Short Term Memory networks (LSTM), Recurrent Neural Network (RNN), and Gated Recurrent Units (GRU) to quantify the intensity of pandemic for the near future. Authors consider the time variable and data nonlinearity when employing neural networks. Each model's

Fadi Al-Turjman

fadi.alturjman@neu.edu.tr

1 Near East University, Nicosia, Mersin 10, Turkey salient features have been evaluated to foresee the number of COVID-19 cases in the next 10 days. The forecasting performance of employed deep learning models shown up to July 01, 2020, is more than $90 \%$ accurate, which shows the reliability of the proposed study.

In the next article with the title "An automatic detection system of diabetic retinopathy using a hybrid inductive machine learning algorithm," it aims to develop a reliable DR screening and detection model to reduce the risk of DRrelated blindness. DR-infected eyes are described by the ophthalmologist for further examination and diagnosis might reduce the risk of vision loss and provide timely and accurate diagnostic information. Hence, this paper proposes a hybrid inductive machine learning algorithm (HIMLA) as an automated DR detection diagnostic tool. The proposed algorithm comprises four stages: pre-processing, segmentation, feature extraction, and classification. At the pre-processing stage, colored fundus images are normalized to a specific brightness level to enhance the quality of the images. In the segmentation stage, the processed image is encoded and decoded to segment the images for improving image quality. Furthermore, feature extraction and classification are performed using multiple instance learning (MIL). The proposed method was evaluated on CHASE datasets for the detection of DR. The accuracy, sensitivity, and specificity of the proposed approach are $96.62 \%, 95.31 \%$, and $96.88 \%$, respectively. These results indicate that HIMLA outperforms other DR models, such as ML-based neovascularization detection in the optic disc (MLB-NVD), genetic algorithm-based diabetic retinopathy (GAB-DR), DL algorithm diabetic retinopathy (DLA-DR), and diagnostic assessment-based DL for diabetic retinopathy (DAD-DR ), which reduces the risk of vision loss.

The fourth article titled "The early warning research on nursing care of stroke patients with intelligent wearable devices under COVID-19," combines regression models and SVM to build a smart wearable device system and builds a system prediction module to predict the patient care needs. By comparing the results of nursing intervention experiments, we 
can see that the smart wearable device designed in this paper has a certain effect in stroke care. Human gait analysis is a well-defined technique for human identification and tracking based on the walking style. It plays an important role in the video surveillance, medical, and defense applications. To effectively reconcile these innovations in medical profession, a new system is required to identify the most important body features which have an impact on an accurate diagnosis and classification. The paper titled "Hybrid FOW - a novel whale optimized firefly feature selector for gait analysis," proposes a novel method FOW which is intended to choose the best gait features as an optimization strategy based on the hybrid integration of whale and firefly algorithms. This approach is utilized for approximating the performance of different classification benchmarks in order to have an efficient medical diagnosis system. Experimentation has been carried out for 35 individuals in which 16 features have been recorded and analyzed.

Due to an unexpected and huge increasing number of patients during the coronavirus (novel COVID-19) pandemic, it is considerably indispensable to monitor patients' health condition continuously before any serious disorder or infection occurs. In transferring the huge volume of produced sensitive health data of patients who do not want their private medical information to be revealed, dealing with security issues of IoT data is a major concern and is a challenging problem that has remained yet. Encountering this challenge, in the paper titled "A secure remote health monitoring model for early disease diagnosis in cloud-based IoT environment," a remote healthmonitoring model that applies a lightweight block encryption method for provisioning security for health and medical data in cloud-based IoT environment is presented. In this model, the patients' health statuses are determined via predicting critical situations through data-mining methods for analyzing their biological data sensed by smart medical IoT devices in which a lightweight secure block encryption technique is used to ensure that the patients' sensitive data become protected. Lightweight block encryption methods have a crucial effective influence on this sort of systems due to the restricted resources in IoT platforms. Experimental outcomes show that the K-star classification method achieves the best results among RF, MLP, SVM, and J48 classifiers, with an accuracy of $95 \%$, precision of $94.5 \%$, recall of $93.5 \%$, and $f$-score of $93.99 \%$. Therefore, regarding the attained outcomes, the suggested model is successful in achieving an effective remote health-monitoring model assisted by secure IoT data in cloudbased IoT platforms.

Nowadays, the biomedical and clinical domains widely use the electromyography (EMG) signal due to its ability to differentiate various neuromuscular diseases. In general, nerves or muscles and the spinal cord influence numerous neuromuscular disorders. The clinical examination plays a major role in early finding and diagnosis of these diseases; the paper titled
"Prediction of muscular paralysis disease based on hybrid feature extraction with machine learning technique for COVID-19 and post-COVID-19 patients" has mainly focused on the prediction of muscular paralysis using EMG signals. Machine learning-based diagnosis of the diseases has been widely used due to its efficiency, and the hybrid feature extraction (FE) methods with deep learning classifier are used for the muscular paralysis disease prediction. The discrete wavelet transform (DWT) method is applied to decompose the EMG signal and reduce feature degradation. The proposed hybrid FE method consists of Yule-Walker, Burg's method, Renyi entropy, mean absolute value, min-max voltage FE, and other 17 conventional features for the prediction of muscular paralysis disease. The hybrid FE method has the advantage of extracting the relevant features from the signals, and the Relief-F feature selection (FS) method is applied to select the optimal relevant feature for the deep learning classifier. The experimental work shows that the proposed hybrid FE method achieved $88 \%$ precision, while the existing neural network $(\mathrm{NN})$ achieved $65 \%$ precision, and the support vector machine (SVM) achieved 35\% precision on the whole EMG signal.

On the other hand, the paper titled "Study on the evolution of information sharing strategy for users of online patient community" discusses the evolution law of information sharing behavior in online health community to study the influence of the different behaviors on health information sharing results and explore ways for information sharing enhancements. Based on the BA scale-free network (Albert-László Barabás and Réka Albert scale-free network), this paper established an information sharing behavior model for members of online health community with the evolutionary game theory method, and discussed the influence of different game parameters and initial conditions on the evolution results of information sharing behavior of community patients with the method of numerical experiment. It is found that the key to improve the level of community information sharing is to improve the benefit of patients' information sharing, the proportion of patients sharing information at the initial moment, and the degree of network nodes, and reduce the sharing cost. Community managers should improve the information conversion ability and information absorption ability of community patients through offline activities, professional guidance, and other forms. At the same time, it can reduce the difficulty and risk of information sharing and strengthen the connection among members, thus comprehensively enhancing the value of the community. With the continuous progress in the era of big data, artificial intelligence technology has begun to get more and more applications in medicine, and it is becoming more and more possible to use artificial intelligence in the diagnostic technology of digestive endoscopy images. The article titled "Artificial intelligence technology based on deep learning in digestive endoscopy imaging diagnosis" mainly 
studies the progress of artificial intelligence technology in the diagnosis of gastrointestinal endoscopy. The main purpose is to add new vitality to the medical conditions that are not yet perfect. This article mainly uses digestive endoscopy to carry out a comprehensive examination of all aspects of the digestive tract under the conditions of accuracy, clarity, and other advantages. Randomly, it selects 14 of the 50 registered patient images for corresponding gastrointestinal endoscopy. The premise is that the subjects must be tested under the same conditions. The ordinary manual detection technology adds 4 groups as the control group, and the start time of the test is the same; when conducting the experiment, the position of each group to be tested must be the same and the detection area is large enough. Finally, image simulations have been carried out. Results show that the sensitivity of artificial intelligence used in the actual experiment is $95.1 \%$, of which the most prominent data value is $97.6 \%$. The accuracy of the digestive endoscopy is $96.6 \%$. The sharpness of the image detected by artificial intelligence is also superior.

In the paper titled "Enhanced BB84 quantum cryptography protocol for secure communication in wireless body sensor networks for medical applications," authors look into critical security threats: secure key distribution. While sharing the secret key between communicating parties in the wireless body sensor networks in the conventional method like via phone or email, the attackers will catch the private key. They can decrypt and modify sensitive medical data. This can cause a significant effect like the patient death. Therefore, we need an effective, secure key distribution scheme for the transmission of human body health-related data to medical professional through wireless links. Accordingly, a new enhanced BB84 quantum cryptography protocol is proposed in this paper for sharing the secret key among communicating parties in a secure manner using quantum theory. Based on experimental results, it is proven that the proposed algorithm EBB84 provides a high secure key distribution method without directly sharing the secret key. It used the quantum mechanism and the bitwise operator for generating and distributing the secret key value to communicating parties for sensitive information sharing in the wireless body sensor networks. The paper titled "The value of artificial intelligence and imaging diagnosis in the fight against COVID19 " uses artificial intelligence-assisted medical imaging diagnosis. It introduces an intelligent COVID-19 detection system and uses it to achieve COVID-19 disease screening and lesion evaluation. The used CT examination has the advantages of fast speed and high accuracy, which can provide a favorable basis for clinical diagnosis. This article collected 32 lung CT scan images of patients with confirmed COVID-19. Two professional radiologists analyzed the CT images using traditional imaging diagnostic methods and artificial intelligenceassisted imaging diagnostic methods. The comparison showed the gap between the two methods. According to experiments,
CT imaging diagnosis assisted by artificial intelligence only takes 0.744 min on average, which can save a lot of time and cost compared with the average time of 3.623 min for conventional diagnosis. In terms of comprehensive test accuracy, it can be concluded that the combination of artificial intelligence and imaging diagnosis has extremely high application value in COVID-19 diagnosis.

In the paper titled "Analyzing gene polymorphism and metal folic acid interactions in neural tube defects using optimized deep recurrent neural networks," authors designed and developed a grey wolf optimizer-assisted deep recurrent neural network to predict the association between gene polymorphism and folic acid interaction in neural tube defects (NTD). The information of different control families and nuclear family information was collected to examine the process of polymorphism. Moreover, the homozygous mutant-type (GG) genotype mothers and folic acid consumption levels were continuously analyzed to identify the link with and risk of NTD. The simulation analysis was performed to evaluate the statistical results in comparison with those obtained using conventional methods. Given the importance of interactions and associations, this research discusses the optimized deep recurrent neural network-based polymorphism process to identify the NTD risk factors with a lower error ratio of $0.015 \%$. Authors found that MFC-GG genotype polymorphism and folic acid consumptions are essential and effective in lowering the prevalence of NTD among offspring with maximum accuracy rate of $99.5 \%$.

Moreover, COVID-19 has caused a serious impact on the global economy. The popularity of mobile shopping makes shopping behavior no longer limited by time and space, so impulse purchase is more commonly seen nowadays; it can effectively promote residents' consumption. However, consensus has not been reached regarding how impulse purchase emerges as a phenomenon, and thus making it difficult to promote consumers' purchase behavior. The article titled "Research on mobile impulse purchase intention in the perspective of system users during COVID-19" aims to explore the generation process of consumers' impulsive purchase intention during the COVID-19 outbreak from the perspective of system users. For this purpose, the research proposes three mobile situation factors: personalized recommendation, visual appeal, and system usability. They have a positive impact on impulse purchase intention by influencing perceived arousal and perceived enjoyment. The experimental method is used for data collection and hypothesis testing. All the hypotheses are supported. And the theoretical value of the model of "mobile environment stimulation-consumer emotion-impulse purchase intention" is confirmed. Based on the conclusion, management suggestions are proposed for mobile shopping merchants from the perspective of improving consumers' shopping experience and expanding 
marketing. Meanwhile, the novel coronavirus pneumonia in 17 city (Hubei) provinces was analyzed by using the principle of thermodynamics in the article titled "Thermodynamic imaging calculation model on COVID-19 transmission and epidemic cities risk level assessment - data from Hubei in China." A thermodynamic imaging model of infectious diseases was established to calculate the cumulative superimposed density of epidemic in 17 Chinese cities (prefectures). An evaluation rule of urban risk grade has been established in order to evaluate the COVID-19 risk in these 17 cities. The results show that (1) the higher the superimposed density of urban epidemic, the more infected people. (2) In the incubation stage, the thermodynamic imaging shows a point distribution, random walk, and outward diffusion trend. In the initial stage, the color of thermodynamic imaging gradually deepened and the range gradually expanded. During the burst stage, the thermodynamic imaging color deepens rapidly and the scope expands rapidly. In the stable stage, the thermodynamic imaging color becomes the darkest and the range is extended to the pole. (3) According to the situation of COVID-19 transmission in Hubei Province, the cumulative superimposed density of the Wuhan epidemic is far more than 10,000, ranking as the "highest risk."

Acknowledgements We are thankful to the anonymous editors/reviewers for their effort in reviewing the above manuscripts. We are also very thankful to the editorial office and the Editor-in-Chief, for their supportive guidance during the entire process.

Publisher's note Springer Nature remains neutral with regard to jurisdictional claims in published maps and institutional affiliations. 